observed, it does not show up precisely where expected. Indeed, rather than appearing at 5/2 (and at the particle-hole conjugate filling fraction 7/2) in the second Landau level, the even denominator emerges, with the application of an in-plane field, at a filling fraction of $3 / 2$ in the lowest Landau level and at 9/2 in the third Landau level. This unusual finding is attributed to the larger ratio of Zeeman-to-cyclotron energies in $\mathrm{ZnO}$ compared with GaAs, such that a band crossing makes the lowest Landau level in the oxide structure resemble the second Landau level (and likewise for the third) in GaAs (Fig. 1). Though seemingly straightforward, this observation provides the first confirmation of the necessary role played by the orbital wavefunction in stabilizing the $5 / 2$ state. Moreover, the ability to tune the system by simply varying the Zeeman energy presents a new opportunity to further explore the complete phase space of the $5 / 2$ state.

Another surprise of the study is the observation that for a purely perpendicular magnetic field, the $7 / 2$ state is robust, whereas the $5 / 2$ state is conspicuously absent ${ }^{2}$. Owing to the inability to vary the density in these high-mobility oxide samples, the $5 / 2$ and $7 / 2$ filling fractions appear at different magnetic fields, where additional terms such as exchange enhancement may be very different. Developing the capability to dynamically vary the density, for example by field-effect gating, will likely be required to fully unravel some of these details. This capability would additionally provide a further opportunity to more fully map the phase diagram of the even-denominator states in these samples compared with what has been possible for GaAs.

Currently the most widely accepted explanation for the 5/2 FQHE is the Moore-Read Pfaffian description ${ }^{8}$, in which an attractive interaction leads to a type of superconducting state of composite fermions. Experimentally, no smoking-gun evidence of this candidate wavefunction has so far been provided. However, some of the salient features have been confirmed, such as the spin-polarized nature of the ground state $e^{9,10}$ and the predicted $e / 4$ fractional charge of the corresponding quasiparticle excitations ${ }^{11,12}$. Perhaps the most intriguing feature of the Pfaffian is the prediction that the corresponding quasi-particles are non-Abelian and therefore could be exploited to realize a type of fault-tolerant quantum computation. At the fundamental level, the Pfaffian describes a $p$-wave quasiparticle pairing with zero-energy Majorana excitation modes. In this way, the discovery of the $5 / 2$ state in oxide heterostructures provides exciting new possibilities for the pursuit of Majorana fermions.

Cory R. Dean is in the Department of

Physics, Columbia University, New York,

New York 10027, USA.

e-mail: cdean@phys.columbia.edu

References

1. Tsui, D. C., Stormer, H. L. \& Gossard, A. C. Phys. Rev. Lett. 48, 1559-1562 (1982)

2. Falson, J. et al. Nature Phys. 11, 347-351 (2015)

3. Laughlin, R. B. Phys. Rev. Lett. 50, 1395-1398 (1983).

4. Haldane, F. D. M. Phys. Rev. Lett. 51, 605-608 (1983).

5. Halperin, B. I. Phys. Rev. Lett. 52, 1583-1586 (1984).

6. Jain, J. K. Phys. Rev. Lett. 63, 199-202 (1989).

Willett, R. et al. Phys. Rev. Lett. 59, 1776-1779 (1987).

8. Moore, G. \& Read, N. Nucl. Phys. B 360, 362-396 (1991).

9. Tiemann, L., Galez., G., Kumada, N. \& Muraki, K. Science 335, 828-831 (2012).

10. Stern, M. et al. Phys. Rev. Lett. 108, 066810 (2012).

11. Dolev, M., Heiblum, M., Umansky, V., Stern, A. \& Mahalu, D. Nature 452, 829-834 (2008).

12. Radu, I. P. et al. Science 320, 899-902 (2008).

\title{
MICROFLUIDICS
}

\section{Mix mastery}

A pair of miscible liquids, some nice clean glass and a Sharpie: that's all it really takes to build your own microfluidic machines. Nate Cira and co-workers have devised a number of tiny lo-fi games for droplets (http://youtu.be/K8Wx2PHIYGI) - and they can even tell you the physics behind them (Nature http://doi.org/2zv; 2015)

Getting droplets to move autonomously on glass isn't new in itself. Surface-energy gradients can be engineered to induce droplet motion, but the techniques are tricky, the gradients required large. Cira et al. found a way to create motile droplets without resorting to complicated surface-preparation protocols. They noticed that droplets made from a mixture of water and propylene glycol didn't spread out like their pure counterparts. The water, being more volatile, evaporated faster. And as evaporation occurred more readily at the edge of the droplet, the concentration of propylene glycol was higher there. This set up a surface-tension gradient, which pulled the liquid towards the centre along the top of the droplet and slowed down spreading.
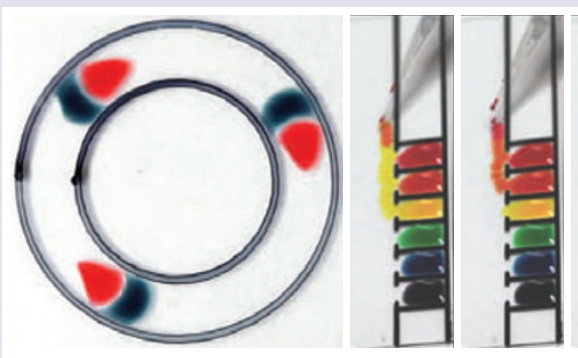

But the constant contact angle was only one piece of the puzzle. The droplets exhibited characteristics of both wetting and non-wetting liquids. They didn't spread readily, but the authors also noticed that they were sitting on a thin fluid film. This meant that they were effectively shielded from the surface - as well as any inhomogeneities that might cause pinning.

Without pinning, the droplets were free to roam. And roam they did - frequently towards one another. Cira et al. observed long-range interactions that were induced by gradients in the water vapour produced by neighbouring droplets. The gradient increased the local relative humidity, slowing evaporation in the thin film and boosting

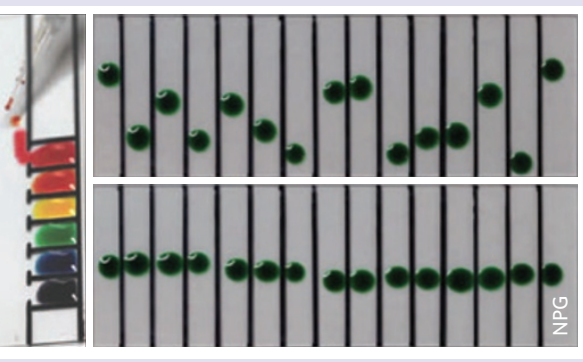

the energy of the liquid-vapour interface, effecting a net movement. Once the droplets were close enough, they coalesced.

And that's when the fun started: Cira etal. used a Sharpie marker to make the glass superhydrophobic - confining the droplets to certain configurations. By playing with the short- and longrange forces, they were able to engineer sustained droplet chasing (left), sorting (middle) and self-organization (right).

Games aside, the technique may provide a physical analogue to help us understand the migration of keratocytes and other motile cells.

ABIGAIL KLOPPER 